\section{Transferrin PET project}

\section{By Michael J. Haas, Senior Writer}

A Memorial Sloan-Kettering Cancer Center team has shown that a transferrin-based PET imaging agent detected prostate cancer in mice with greater sensitivity than ${ }^{18} \mathrm{~F}$-labeled fluorodeoxyglucose. ${ }^{1}$ The new agent also detected a precancerous prostate condition that is not amenable to existing imaging technologies. The team now plans to take the agent into Phase I testing to detect prostate cancer.

PET imaging uses an agent labeled with a positron-emitting radionuclide. ${ }^{18} \mathrm{~F}$-labeled fluorodeoxyglucose (FDG) is the most widely used PET agent for tumor imaging because cancer cells have higher glucose uptake than normal cells and ${ }^{18} \mathrm{~F}$ can be readily incorporated into glucose without significantly altering the biochemical properties of the sugar.

Another compound that could serve as the basis for a PET imaging agent is transferrin, a plasma protein that binds iron and carries it into cells via transferrin receptor protein 1 (TFRC; TFR; CD71). Most tumor types express higher levels of TFRC than normal cells, and as a result multiple studies over the past 30 years have explored the tumor-imaging potential of PET agents based on transferrin labeled with ${ }^{18} \mathrm{~F},{ }^{45} \mathrm{Ti},{ }^{97} \mathrm{Ru}$ or ${ }^{131} \mathrm{I}^{2-5}$

However, transferrin-based PET agents and FDG share a key limitation: rapid metabolism. The resulting radiolabeled metabolites can accumulate in normal tissues and produce background signals that make it difficult to obtain clear images of suspect tissues.

This has been especially problematic for imaging the prostate because many metabolites of FDG and transferrin-based agents can accumulate in the nearby bladder.

A way around the prostate-imaging problem emerged a few years ago from a pair of studies by the Memorial Sloan-Kettering team. In 2009, the group reported that desferrioxamine (DFO) labeled with the positron-emitting radionuclide ${ }^{89} \mathrm{Zr}$ was stable in ex vivo human serum for up to seven days and produced high-resolution PET images in healthy mice. ${ }^{6}$

In 2010, the team showed that ${ }^{89} \mathrm{Zr}$-DFO conjugated to an antibody against prostate-specific membrane antigen (PSMA; FOLH1; GCPII) was metabolically stable and produced high-contrast PET images of xenograft prostate tumors in mice. ${ }^{7}$

These findings led the team to postulate that ${ }^{89} \mathrm{Zr}$-DFO conjugated to transferrin might also be stable and thus more broadly useful as a tumor-imaging agent than the ${ }^{89} \mathrm{Zr}$-DFO-antibody conjugate.

First, the team showed that conjugates of ${ }^{89} \mathrm{Zr}$-DFO and human transferrin were metabolically stable in ex vivo human blood for up to four days. Similarly, conjugates using mouse transferrin were stable for the same duration in the circulation of healthy mice.

The team also confirmed the conjugates' mode of action by showing uptake in mouse and human cancer cell lines expressing c-Myc (MYC) - a transcription factor that upregulates TFRC.

For its subsequent experiments with ${ }^{89} \mathrm{Zr}$-DFO-transferrin, the team focused on prostate tumors, because these presented the greatest challenge to existing PET agents.

The group showed that PET imaging with ${ }^{89} \mathrm{Zr}$-DFO-transferrin could distinguish between xenograft prostate tumors in mice with differing levels of TFRC activity. In addition, the agent detected high-grade prostatic intraepithelial neoplasia (PIN) in four-monthold mice-about eight months before these animals develop prostate tumors-whereas FDG-PET did not.

PIN is thought to be a precursor to prostate cancer but is currently detectable only by histological analysis. Thus, it is usually found in biopsies taken from patients already suspected of having prostate cancer.

A key advantage of ${ }^{89} \mathrm{Zr}-\mathrm{DFO}-$ transferrin is its metabolic stability, team coleader Jason Lewis told SciBX. "This allowed us to quantify fairly modest changes in MYC activity and TFRC expression to image tumors in a region of the animal that has been notoriously challenging to image," he said.

Another advantage is the long half-life of ${ }^{89} \mathrm{Zr}$, which allows
"This allowed us to quantify fairly modest changes in MYC activity and TFRC expression to image tumors in a region of the animal that has been notoriously challenging to image."

- Jason Lewis,

Memorial Sloan-Kettering Cancer Center "imaging over time spans far

more appropriate for capturing the pharmacology of long-circulating molecules like transferrin" than what was possible with other transferrin-based PET imaging agents, he said.

Lewis is vice chair for basic research, chief attending radiochemist and director of the cyclotron core in the Department of Radiology at Memorial Sloan-Kettering. He also holds a joint appointment in the Sloan-Kettering Institute's molecular pharmacology and chemistry program.

Charles Sawyers, chair of the human oncology and pathogenesis program at Memorial Sloan-Kettering and investigator at the Howard Hughes Medical Institute, co-led the team.

Data were reported in Nature Medicine. ${ }^{1}$

\section{Aggressive images}

Despite the ability of ${ }^{89} \mathrm{Zr}$-DFO-transferrin to detect PIN, the agent probably would not find utility as a tool to screen for the risk of prostate cancer because "there is no unmet clinical need to detect and image PIN," Lewis said.

Instead, he said, the agent could be used to distinguish between indolent and aggressive prostate cancer at the time of diagnosis because 


\section{ANALYSIS}

existing tools_-such as levels of prostate-specific antigen (KLK3; PSA) are not reliable predictors of aggressive cancer.

"We know aggressive disease can be MYC-driven, and it's likely that all tumors with MYC amplification will be aggressive," he said. "So ${ }^{89} \mathrm{Zr}$-DFO-transferrin could be used to profile patients according to MYC status" and identify those whose cancer is likely to be aggressive.

However, Lewis noted that ${ }^{89} \mathrm{Zr}$-DFO-transferrin would not be strictly limited to detection of MYC-dependent cancers because other proteins and pathways can also upregulate TFRC. As an example, he cited the team's study of the agent in xenograft models of glioblastoma multiforme (GBM) in which TFRC is upregulated by aberrant phosphoinositide 3-kinase (PI3K) signaling. Those results are in press with the Journal of Nuclear Medicine, he said.

He declined to disclose whether the team has tested ${ }^{89} \mathrm{Zr}$-DFOtransferrin in mice with additional tumor types, but he said there are many good animal models of MYC-dependent-and thus TFRCexpressing-lymphoma and other cancers in which the PET agent could be tested.

The team is now running IND-enabling studies of ${ }^{89} \mathrm{Zr}$-DFO-transferrin and is raising funds for a Phase I trial of the agent in patients with prostate cancer that it expects to begin in about a year, he said.

Memorial Sloan-Kettering spokesperson Andrea Molinatti declined to disclose who will fund the trial because that funding is under review by the NIH, she said.
Lewis added that the team is starting with prostate cancer because the "abundance of patients coming through Memorial Sloan-Kettering for treatment" will make trial enrollment easy. After completing the prostate cancer trial, the team plans to test ${ }^{89} \mathrm{Zr}$-DFO-transferrin in patients with lymphoma, he said.

Lewis said the findings reported in Nature Medicine are unpatented.

Haas, M.J. SciBX 5(39); doi:10.1038/scibx.2012.1022

Published online Oct. 4, 2012

\section{REFERENCES}

1. Holland, J.P. et al. Nat. Med.; published online Sept. 23, 2012; doi: $10.1038 / \mathrm{nm} .2935$

Contact: Jason S. Lewis, Memorial Sloan-Kettering Cancer Center, New York, N.Y.

e-mail: lewisj2@mskcc.org

Contact: Charles L. Sawyers, same affiliation as above e-mail: sawyersc@mskcc.org

2. Som, P. et al. Eur. J. Nucl. Med. 8, 491-494 (1983)

3. Prost, A.C. et al. Int. J. Rad. Appl. Instrum. B 17, 209-216 (1990)

4. Aloj, L. et al. J. Nucl. Med. 40, 1547-1555 (1999)

5. Vavere, A.L. \& Welch, M.J. J. Nucl. Med. 46, 683-690 (2005)

6. Holland, J.P. et al. Nucl. Med. Biol. 36, 729-739 (2009)

7. Holland, J.P. et al. J. Nucl. Med. 51, 1293-1300 (2010)

COMPANIES AND INSTITUTIONS MENTIONED

Howard Hughes Medical Institute, Chevy Chase, Md. Memorial Sloan-Kettering Cancer Center, New York, N.Y. National Institutes of Health, Bethesda, Md.

Sloan-Kettering Institute, New York, N.Y. 